\title{
A coaxial-slot antenna for invasive microwave hyperthermia therapy
}

\author{
Teng Jiao", Hua Wang", Yang Zhang", Xiao Yu, Huijun Xue, Hao Lv, Xijing Jing, Hua Zhan, \\ Jianqi Wang*
}

Department of Biomedical Engineering, The Fourth Military Medical University, Xi'an, China

Email: *jiaoteng@,fmmu.edu.cn

Received 22 December 2011; revised 29 January 2012; accepted 17 February 2012

\begin{abstract}
When the technique of invasive microwave hyperthermia is applied to cancer treating, the distribution of microwave thermal field and the effect of the therapy are determined by the type of microwave radiative antenna. The thermal field of biological tissue produced by microwave radiative antenna is investigated in this paper. The distribution of thermal field and specific absorption rate (SAR) of invasive coaxial-slot antenna in the frequency of $2450 \mathrm{MHz}$ are obtained by the technique of finite element analysis. According to the experiment of heating the ex vivo pork liver by this kind of antenna, the result concordant with the theory is obtained. Therefore, it is suggested that this research could be a reference for clinical therapy and operation scheme.
\end{abstract}

Keywords: Microwave Hyperthermia Therapy; Antenna; Thermal Field; SAR

\section{INTRODUCTION}

Recent years, invasive microwave hyperthermia for cancer treating has been widely investigated. In the research of the biological effects of microwave and microwave therapy, the radiator, which is also called antenna, is used to radiate the electromagnetic energy of the microwave oscillator to the exposed biosystem spatially or couple the biosystem directly.

When the technique of invasive microwave hyperthermia is applied to cancer treating, the distribution of microwave thermal field is determined by the type of microwave radiative antenna. In the microwave mesenchymal thermal therapy, the radiative antenna is linear antenna, which portrait length is far more than transverse diameter. The invasive system distribution energy obtained from the thermotherapic probe or duct's energy

\footnotetext{
*Corresponding author.

\#The first three authors contributed equally to this work and should be regarded as co-first authors.
}

source of powerful small antenna. Invasive microwave radiative antenna is different from other antennas which are utilized on the humanbody's superficial surface. And it should be smaller volume, stronger radiative capability and considerable intensity. Due to such advantages as direct exposure into the tissue of pathological changes, high efficiency of heating and easy control of power et $a l$., it has been largely utilized clinically.

The performance of antenna can be determined by simulating tumorous SAR. Concretely, it is calculated by three dimension data model as EFIE and MFIE. Jaehoon et al. inserted an antenna into the human body and calculated the distribution of SAR by FDTD [1]. Zouheir et al. developed an electrothermal cordis duct model to distinguish RF and microwave energic transmission [2]. Monoantenna made by coaxial wire was inserted into myocardium for heating, and SAR was calculated by finite element modeling. Mikaya et al. established the precise model of antenna by GBM [3]. Francesca Rossetto calculated the distribution of SAR of $915 \mathrm{MHz}$ microwave antenna by FDTD and simulated on three different human tissues that is of importance to clinic [4]. Whayne et al. investigated $915 \mathrm{MHz}$ and $2450 \mathrm{MHz}$ Monoantenna and did some microwave ablation experiments on cardiac muscle in vitro [5]. Rosenbaum et al. obtained some optimum frequency and pattern of antenna in way of heating phantoms by helical and whip shape antennas [6-8].

In order to investigate the distribution of thermal field and optimum antennal pattern in invasive microwave thermal therapy better, the invasive coaxial-slot antenna in the frequency of $2450 \mathrm{MHz}$ was design. After investigating the thermal field of biological tissue which induced by microwave radiative antenna, the distribution of thermal field and SAR of invasive coaxial-slot antenna in the frequency of $2450 \mathrm{MHz}$ were obtained by the technique of finite element analysis. What's more, the $2450 \mathrm{MHz}$ HDZ-1 intelligent multifunctional microwave oncotherapic device was used to do heating experiment on pork liver ex vivo, and a contrast and discussion be- 
tween simulative results and experimental results was made.

\section{ANTENNA DESIGN}

That a radiative slot is opened in some distance from the top of $50 \Omega$ coaxial wire and slot point is exposed from the media layer. In addition, exterior and interior conductor is connected on the top of the antenna. The structure of coaxial-slot antenna and its size and parameter were shown in Figure 1.

\section{RESULTS AND DISCUSSION}

Early in year 1948, Pennes proposed the famous biological conduct heat equation, which demonstrate the balance between heat conduction of perfused tissues, heat loss from blood flow, metabolic heat from heating and energy deposition. For a long time, this equation has showed its metal in tumorous thermotherapy, blood

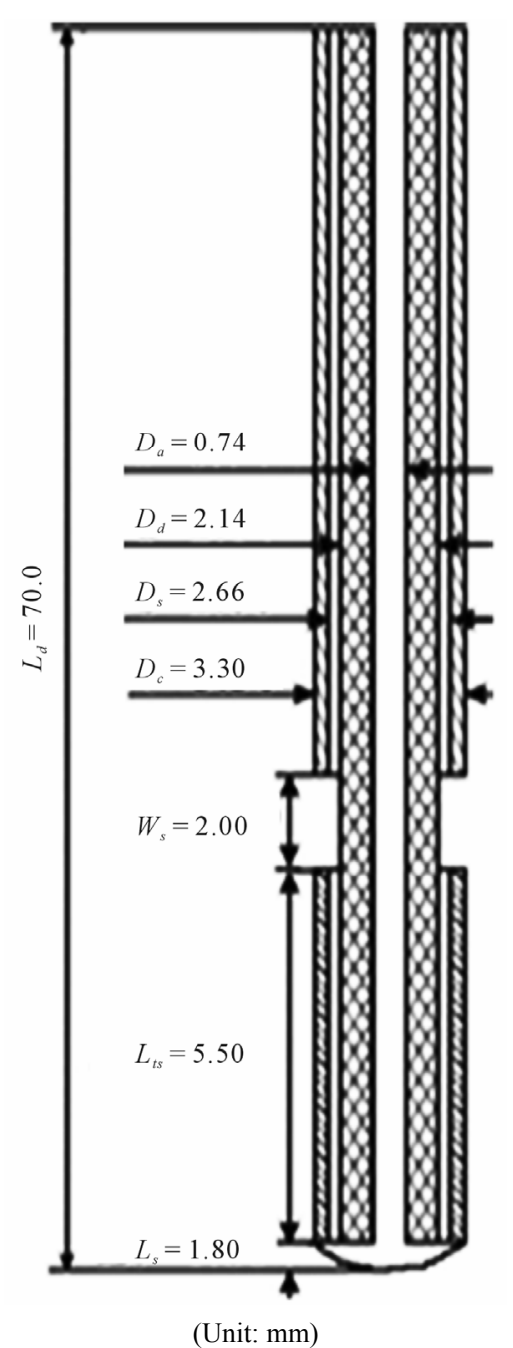

Figure 1. The structure, size and parameter of coaxial-slot antenna. circulation inhibition, clinical thermal science such as freezing and empyrosis and so on. Because of simplicity and applicability, it is the most popular model in biology conduct heat domain as well. Its equation format is:

$$
\rho C \frac{\partial T}{\partial t}=\nabla \cdot \kappa \nabla T+\omega_{b} \rho_{b} C_{b}\left(T_{a}-T\right)+Q_{i}
$$

$T_{a}$ is proper artery temperature, and usually be hypothesized as core temperature (about $37^{\circ} \mathrm{C}$ ).

$\omega_{b} \rho_{b} C_{b}\left(T_{a}-T\right)$ is item of heat transfer due to hemoperfusion called $Q_{b}$. This equation can also write as:

$$
\rho C \frac{\partial T}{\partial t}=\nabla \cdot \kappa \nabla T+\omega_{b} \rho_{b} C_{b}\left(T_{b}-T\right)+Q_{m e t}+Q_{e x t}
$$

$\rho$ is tissue density and $\rho_{b}$ is blood density, whose unit is $\mathrm{kg} / \mathrm{m}^{3}$. C is tissue specific heat and $C_{b}$ is blood specific heat, whose unit is $\mathrm{J} / \mathrm{kg} \cdot \mathrm{K} . \kappa$ is tissue heat conductivity, whose unit is $\mathrm{W} / \mathrm{m} \cdot \mathrm{K}, \omega_{b}$ is blood perfusion rate, whose unit is $s-1, T$ is tissue temperature and $T_{b}$ is blood temperature, whose unit is $K . Q_{m e t}$ is metabolic heat and $Q_{e x t}$ is absorbing microwave radiation energy per tissue unit, whose unit is $\mathrm{J} / \mathrm{m}^{3} \cdot \mathrm{s}$.

In this experiment, we use pork liver ex vivo. Thus, it is no need to consider the effect of blood filling rate when analyzed, and power input is pulse power. So Pennes equation is changed as:

$$
\rho C \frac{\partial T}{\partial t}=\nabla \cdot \kappa \nabla T+Q_{a v} \cdot k(t)
$$

$Q_{a v}$ is impedance heat, which is heat source in heating process. $k(t)$ is power's duty ratio factor, which calls the square function from Matlab in calculation, and expression as follow:

$$
k(t)=[\operatorname{square}(2 \pi \cdot t / 60,100 D)+1] / 2
$$

$D$ is duty ratio, which values as $25 \%(15 \mathrm{~s} / 60 \mathrm{~s})$ or $75 \%(22.5 \mathrm{~s} / 30 \mathrm{~s})$ in this article.

The finite element method was adopted to solve Pennes equation. Antennal duty ratio of output power is $25 \%$. Dielectric characteristic parameter in calculation is introduced in Table $\mathbf{1}$ and model's finite element mesh in Figure 2.

Figures 3 and 4 show the distribution of temperature and SAR after coaxial-slot antenna heating tissue for 240 $\mathrm{s}$ respectively, and coaxial-slot antennal hot area range is about $2.6 \mathrm{~cm} \times 3.5 \mathrm{~cm}$.

Figure 5 is a curve of SAR changes in company with depth of penetration in the antennal external verge (distance $r$ from axis equals $1.66 \mathrm{~mm}$ ).

Make a line tightly near antenna external diameter from antenna axis and $\mathrm{r}=1.66 \mathrm{~mm}$. On this line, SAR variation is showed in Figure 5. There are three peaks in the coaxial-slot antenna, with the hightest one corresponding to top of antenna, other two corresponding to antennal slot. Therefore, radiation is weakest in coaxial- 
Table 1. Pork liver tissue and antennal thermal parameter and dielectric characteristic parameter.

\begin{tabular}{ccc}
\hline parameter & numerus & demonstration \\
\hline$\varepsilon_{r i}$ & 2.03 & Medium layer relative dielectric constant \\
$\varepsilon_{r c}$ & 2.60 & sheath relative dielectric constant \\
$\varepsilon_{r}$ & 43.03 & hepatic tissue relative dielectric constant \\
$\sigma$ & $1.69(\mathrm{~S} / \mathrm{m})$ & hepatic tissue electrical conductivity \\
$\kappa$ & $0.512(\mathrm{~W} / \mathrm{m} \cdot \mathrm{K})$ & hepatic tissue heat conductivity \\
$\rho$ & $1060\left(\mathrm{~kg} / \mathrm{m}^{3}\right)$ & hepatic tissue density \\
$C$ & $3.60 \times 10^{3}(\mathrm{~J} / \mathrm{kg} \cdot \mathrm{K})$ & Hepatic tissue specific heat \\
$\rho_{b}$ & $1000\left(\mathrm{~kg} / \mathrm{m}^{3}\right)$ & blood density \\
$C_{b}$ & $4.18 \times 10^{3}(\mathrm{~J} / \mathrm{kg} \cdot \mathrm{K})$ & density filling rate \\
$\omega_{b}$ & $3.6 \times 10^{-3}\left(\mathrm{~s}^{-1}\right)$ &
\end{tabular}

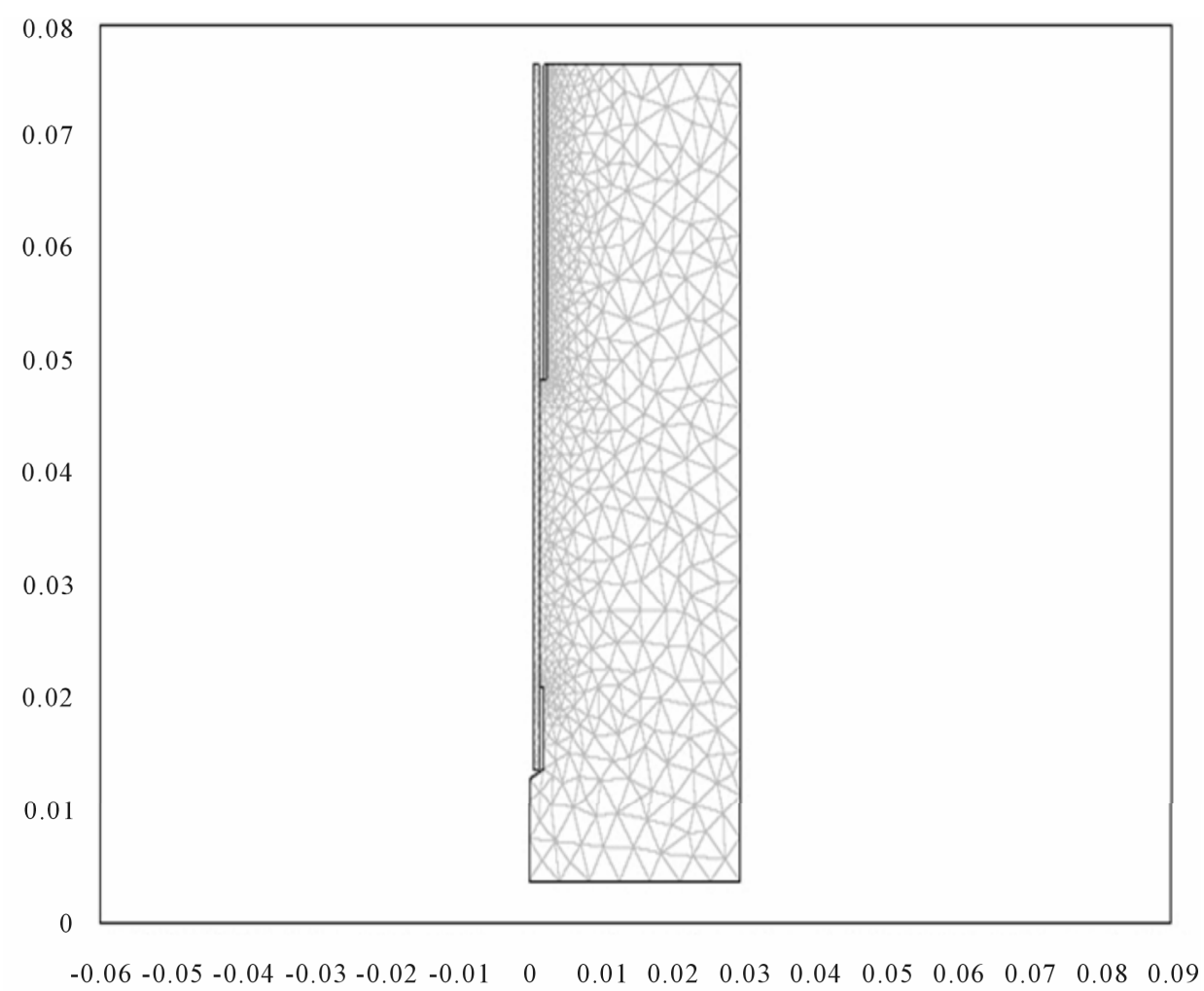

Figure 2. Model's finite element mesh.

slot antennal slot and strongest on top of antenna. In actual thermotherapy, we should correspond antennal strongest radiation point to the middle of tumor tissue to obtain best heating effect.

To investigate the distribution of thermal field and optimum pattern of antenna in invasive microwave thermotherapy better, the $2450 \mathrm{MHz}$ HDZ-1 intelligent multifunctional microwave oncotherapic device was used to carry out ex vivo pork liver experiment. Microwave on- cotherapic device is composed of microwave heating system and thermo-measurement system. Microwave system is composed of four $2450 \mathrm{MHz}$ microwave power source, whose average power is $200 \mathrm{~W}$.

Thermo-measurement system is composed of 8-channel Pt 1000 platinum electric resistance thermo-measurement needle and corresponding magnified and sampling circuit. The diameter of thermo-measure needle is only 2 $\mathrm{mm}$, and it can resist high temperature and acid base and 


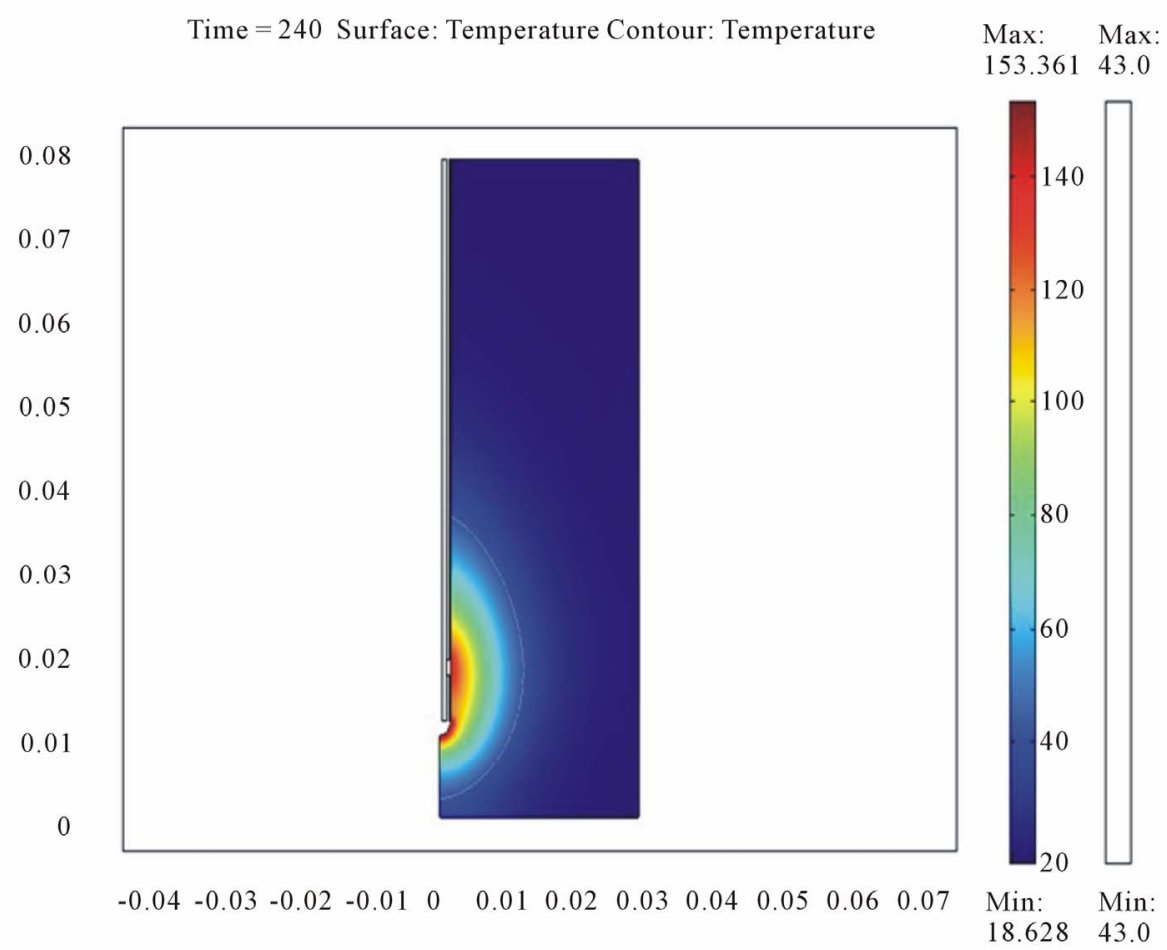

Figure 3. Temperature distribution after heating $240 \mathrm{~s}$.

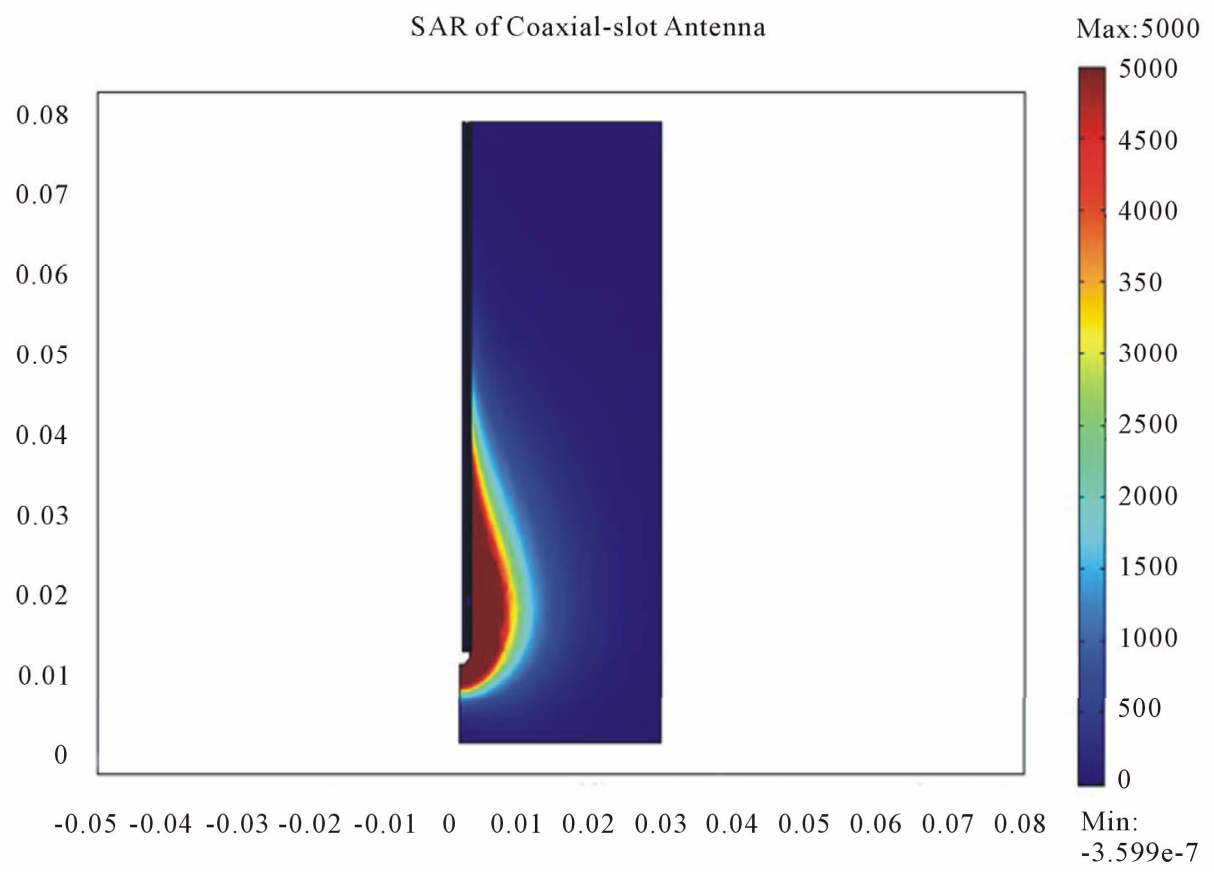

Figure 4. SAR distribution after heating $240 \mathrm{~s}$.

has strong strength. Because it will bring about damage on tissue with this measure method, it limits point amounts of measure temperature. So, we choose only 2 or 3 thermo-measure needles to monitor variation tendency in this experiment but it can not be set up actual temperature distribution diagram. On account of protein denaturation when heating tissue, which shows as proteic coagulation, we can compare coagulation field and simulative denaturation field to check up reliability of results and investigate efficiency of microwave radiometer, offering theorical reference to clinical oncotherapy.

Figure 6 shows cross-section picture of coaxial-slot 


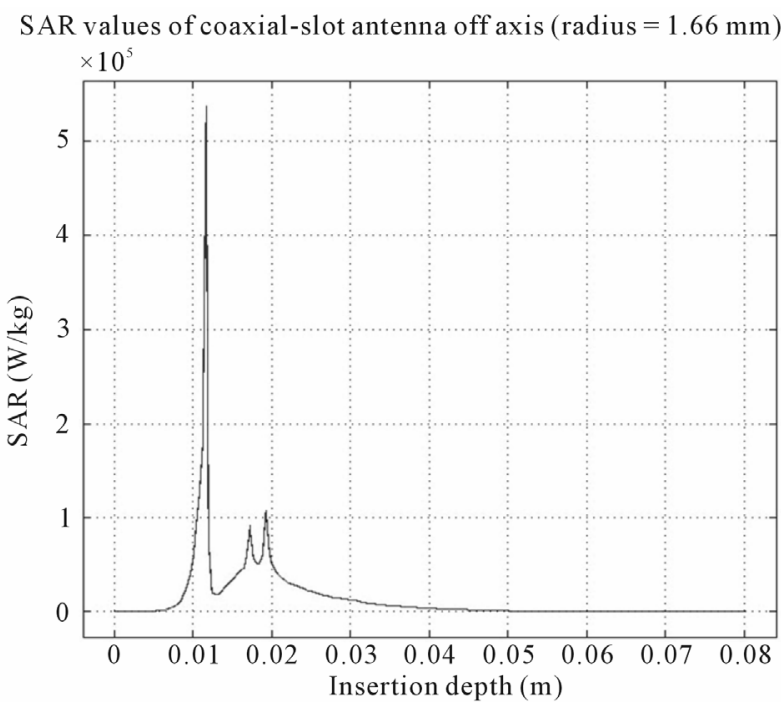

Figure 5. SAR curve in coaxial-slot antenna external diameter.

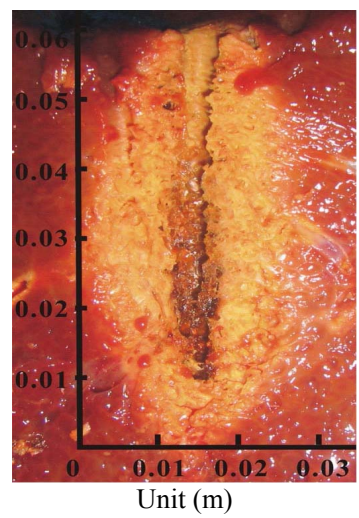

Figure 6. Cross-section picture of coaxial-slot antenna heating pork liver tissue for $240 \mathrm{~s}$.

antenna heating pork liver tissue for $240 \mathrm{~s}$ and hot area range is $2.8 \mathrm{~cm} \times 6.0 \mathrm{~cm}$ after experiment. Temperature distribution is concordant with theoretic result.

\section{CONCLUSIONS}

Therefore, the technology of invasive microwave hyperthermia therapy heat the cancer regions up to 43 degrees and simultaneously make as little damage as possible to normal tissues, finally kill the tumor cells [9].

The antennal simulative results and experimental results show that the entirely temperature distribution of coaxial-slot antenna is homogeneous and this antenna has better heating effect. Even if around the antenna, the top temperature does not exceed $200^{\circ} \mathrm{C}$. So the phenomenon of part hyperpyrexia near the antennas will not occur. And after heating, tissue will not be scorched. Therefore, it is suitable for clinical thermotherapy.

\section{ACKNOWLEDGEMENTS}

This work was supported by the Natural Science Foundation of Shaanxi Province under Award Number 2010K13-02-01.

\section{REFERENCES}

[1] Kim, J. and Rahmat-Samii, Y. (2004) Implanted antennas inside a human body: Simulations, designs, and characterizations. IEEE Transactions on Microwave Theory and Techniques, 52, 1934-1943. doi:10.1109/TMTT.2004.832018

[2] Khebir, A., Kaouk, Z. and Savard, P. (1995) Modeling a microwave catheter antenna for cardiac ablation. Microwave Symposium Digest, IEEE MTT-S International, Orlando, 16-20 May 1995, 299-302. doi:10.1109/MWSYM.1995.406047

[3] Lumöri, M.L.D. (2000) Experimentally based modeling of field sources for three-dimensional computation of SAR in electromagnetic hyperthermia and treatment planning. IEEE Transactions on Microwave Theory and Techniques, 48, 1522-1530. doi:10.1109/22.869003

[4] Rossetto, F. and Staufer, P.R. (1999) Effect of complex bolus-tissue load configurations on SAR distributions from dual concentric conductor applicators. IEEE Transactions on Biomedical Engineering, 46, 1310-1319. doi:10.1109/10.797991

[5] Whayne, J.G., Nathan, S. and Haines, D.E. (1994) Microwave catheter ablation of myocardium in vitro. Circulation, 89, 2390-2395.

[6] Rosenbaum, R.M., Greenspon, A.J., Hsu, S., Walinsky, P. and Rosen, A. (1993) RF and microwave ablation for the treatment of ventricular tachycardia. Microwave Symposium Digest, IEEE MTT-S International, Atlanta, 14-18 June 1993, 1155-1158. doi:10.1109/MWSYM.1993.277076

[7] Wu, J.K. (1994) Two problems of computer mechanics program system. Proceedings of Finite Element Analysis and $C A D$, Peking University Press, Beijing, 9-15.

[8] Saito, K., Taniguchi, T., Yoshimura, H. and Ito, K. (2001) Estimation of SAR distribution of a tip-split array applicator for microwave coagulation therapy using the finite element method. IEICE Transactions on Electronics, E84-C, 948-954.

[9] Yadava, R.L. (2003) RF/microwaves in bio-medical applications. 8th International Conference on Electromagnetic Interference and Compatibility (INCEMIC 2003), Yadava, 18-19 December 2003, 81-85. doi:10.1109/ICEMIC.2003.1287768 